\title{
On Fixed Point Property under Lipschitz and Uniform Embeddings
}

\author{
Jichao Zhang, ${ }^{1}$ Lingxin Bao $(\mathbb{D}),{ }^{2,3}$ and Lili Su ${ }^{4}$ \\ ${ }^{1}$ School of Science, Hubei University of Technology, Wuhan 430068, China \\ ${ }^{2}$ School of Computer and Information, Fujian Agriculture and Forestry University, Fuzhou, 350002, China \\ ${ }^{3}$ Key Laboratory of System and Control, Academy of Mathematics and Systems Science, Academia Sinica, Beijing 100190, China \\ ${ }^{4}$ Engineering University of the Chinese People's, Armed Police Force, Xian 710086, China
}

Correspondence should be addressed to Lingxin Bao; bolingxmu@sina.com

Received 19 June 2018; Accepted 24 September 2018; Published 21 October 2018

Academic Editor: Calogero Vetro

Copyright (C) 2018 Jichao Zhang et al. This is an open access article distributed under the Creative Commons Attribution License, which permits unrestricted use, distribution, and reproduction in any medium, provided the original work is properly cited.

\begin{abstract}
We first present a generalization of $\omega^{*}$-Gâteaux differentiability theorems of Lipschitz mappings from open sets to those closed convex sets admitting nonsupport points and then show that every nonempty bounded closed convex subset of a Banach space has the fixed point property for isometries if it Lipschitz embeds into a super reflexive space. With the application of Baudier-LancienSchlumprecht's theorem, we finally show that every nonempty bounded closed convex subset of a Banach space has the fixed point property for continuous affine mappings if it uniformly embeds into the Tsirelson space $T^{*}$.
\end{abstract}

\section{Introduction}

The infinite Rademacher theorem $[1,2]$ states that every Lipschitz mapping from a separable Banach space to a Banach space with the Radon-Nikodým property (in short, RNP) is almost everywhere Gâteaux differentiable, i.e., everywhere Gâteaux differentiable off an Aronszain null set. It is shown in $[3,4]$ that Aronszajn null sets (introduced by Aronszajn [1]), Gauss null sets (introduced by Phelps [5]), and cube null sets (introduced by Mankiewicz [6]) coincide. In particular, Aronszajn null sets and Lebesgue null sets coincide in a finite dimensional Banach space [3]. To establish the localized version of the infinite Rademacher theorem, Cheng and Zhang [7] substituted closed convex subsets of a Banach space $X$ for $X$ and proved that every Lipschitz mapping from a closed convex subset $C$ of a Banach space with nonempty nonsupport point set $N(C)$ to a Banach space with the RNP is almost everywhere Gâteaux differentiable.

As is well known, dual Banach spaces without separability may fail RNP. The Lipschitz mappings from a separable Banach space to a dual Banach space without separability need not be Gâteaux differentiable anywhere. To circumvent this obstruction, a weaker notion of a $\omega^{*}$-Gâteaux differentiability was introduced (see, for instance, $[3,8]$ ). Heinrich and
Mankiewicz [9] proved the infinite $\omega^{*}$-Rademacher theorem: every Lipschitz mapping from a separable Banach space to the dual space of a separable Banach space is almost everywhere $\omega^{*}$-Gâteaux differentiable. Therefore, a question naturally arises.

Question 1. The question is whether the infinite $\omega^{*}$-Rademacher theorem can be localized to those Lipschitz mappings from a closed convex set $C$ admitting nonempty nonsupport point set $N(C)$ to the dual space of a separable Banach space.

The aim of this paper focuses on the study of the question above. Based on the ideas of the infinite $\omega^{*}$-Rademacher theorem, we shall prove the following theorem.

Theorem 2. Suppose that $C$ is a closed convex set of a separable Banach space $X$ with $N(C) \neq \emptyset$ and that $Y$ is a separable Banach space. Let $f: C \longrightarrow Y^{*}$ be a Lipschitz mapping. Then $f$ is $\omega^{*}$-Gâteaux differentiable off a Aronszajn null set of $N(C)$.

As an application of Theorem 2, we obtain the following result. 
Theorem 3. Every nonempty bounded closed convex subset $C$ of a Banach space $X$ has the fixed point property for isometries if it Lipschitz embeds into a super reflexive space.

With the application of Baudier-Lancien-Schlumprecht's theorem, we finally get the following result.

Theorem 4. Every nonempty bounded closed convex subset $C$ of a Banach space $X$ has the fixed point property for continuous affine mappings if it uniformly embeds into the Tsirelson space $T^{*}$.

The letter $X$ will always be a real Banach space and $X^{*}$ its dual. $S_{X}$ denotes the unit sphere of $X$. For a subset $C \subset X$, we denote by $\bar{C}$ the closure of $C$. For simplicity, we also denote $X_{C}=\overline{\operatorname{span}}(C)$ and $C_{x}=\cup\{\lambda(C-x): \lambda>0\}$ for some $x \in C$.

\section{A Proof of Theorem 2}

We first recall definitions of support points [5, 7] and Aronszajn null sets $[1,3]$.

Definition 5. Suppose that $C$ is a convex set in a Banach space $X$. A point $x \in C$ is said to be a support point of $C$ if there exists a non-zero functional $x^{*} \in X^{*}$ such that $\left\langle x^{*}, x\right\rangle=$ $\max \left\{\left\langle x^{*}, y\right\rangle: y \in C\right\}$.

We denote by $S(C)$ the set of all support points of $C$ and by $N(C)$ the set of all nonsupport points of $C$.

Let $X$ be a Banach space. Let $\lambda$ be the one dimensional Lebesgue measure. For each $x \in X \backslash\{0\}$, set

$$
\begin{aligned}
& \Lambda(x)=\{A \\
& \subset X: A \text { is a Borel set such that } \lambda(A \cap(z+\mathbb{R} x)) \\
& =0 \text { for every } z \in X\},
\end{aligned}
$$

and for a finite or infinite sequence $\left\{x_{n}\right\}$ of nonzero points in $X$, set

$$
\Lambda\left(\left\{x_{n}\right\}\right)=\left\{A=\cup A_{n}, A_{n} \in \Lambda\left(x_{n}\right) \text { for every } n \in \mathbb{N}\right\}
$$

Definition 6. Suppose that $A$ is a Borel set in a separable Banach space $X$. The set $A$ is called an Aronszajn null set (or simply, a null set) if

$$
A \in \cap\left\{\Lambda\left(\left\{x_{n}\right\}\right):\left\{x_{n}\right\}\right.
$$$$
\text { are nonzero vectors such that } \operatorname{span}\left\{x_{n}\right\}
$$

is dense in $X\}$.

Note that $N(C)$ is a $G_{\delta}$ set; hence it is a Borel set (see, for instance, $[7,10])$.

The following two lemmas are presented in $[3,8]$.

Lemma 7. Let $Y$ be a Banach space with the RNP. Then every Lipschitz function $f$ from an open subset $U$ of $\mathbb{R}^{n}$ into $Y$ is Gâteaux differentiable off a null set of $U$.
Lemma 8. Let $F$ be an n-dimensional subspace of Banach space $X$, and let $\left\{x_{k}\right\}_{k=1}^{n}$ be a basis for F. Let $\lambda_{n}$ be the Lebesgue measure on $F$, and let $A$ be a Borel subset of $X$ such that $\lambda_{n}(F \cap(A+x))=0$ for every $x \in X$. Then $\left.A \in \Lambda\left(\left\{x_{k}\right\}_{k=1}^{n}\right\}\right)$.

Definition 9. Suppose that $C$ is a closed convex set of a separable Banach space $X$ with $N(C) \neq \emptyset$ and that $Y$ is a separable Banach space. Let $f: C \longrightarrow Y^{*}$ be a Lipschitz mapping. Then $f$ is said to be $\omega^{*}$-Gâteaux differentiable at $x \in N(C)$ if there exists a bounded linear operator $T_{x}: C \longrightarrow$ $Y^{*}$ such that for every $h \in C_{x}$ and every $y \in Y$ the following limit exists:

$$
T_{x}(h)(y)=\lim _{\lambda \rightarrow 0} \frac{(f(x+\lambda h)-f(x))(y)}{\lambda} .
$$

In this case $T_{x}$ is called the $\omega^{*}$-derivative of $f$ at $x$ and is denote by $D_{f}^{*}(x)$.

Theorem 10. Suppose that $C$ is a closed convex set of a separable Banach space $X$ with $N(C) \neq \emptyset$ and that $Y$ is a separable Banach space. Let $f: C \longrightarrow Y^{*}$ be a Lipschitz mapping; i.e., there exists $K>0$ such that $\|f(x)-f(y)\| \leq$ $K\|x-y\|$ for all $x, y \in C$. Let $\left\{x_{n}\right\}$ be a sequence which consists of linearly independent vectors in $N(C)$. For every $n \in \mathbb{N}$, set $X_{n}=\operatorname{span}\left\{x_{k}: k \leq n\right\}$ and set $\left.f_{n} \equiv f\right|_{C \cap X_{n}}$. Then

(i) $f_{n}$ is $\omega^{*}$-Gâteaux differentiable off a null set of $N(C) \cap$ $X_{n}$ for every $n \in \mathbb{N}$;

(ii) $\left\|D_{f_{n}}^{*}(x)\right\| \leq K$ whenever $D_{f_{n}}^{*}(x)$ exists for every $n \in \mathbb{N}$;

(iii) If, in addition, there exists $k>0$ such that $\| f(x)-$ $f(y)\|\geq k\| x-y \|$ for all $x, y \in C$, then then $D_{f_{n}}^{*}(x): X_{n} \longrightarrow$ $Y^{*}$ is a linear isomorphism for almost every $x \in N(C) \cap X_{n}$.

Proof. (i). Without loss of generality, we assume that $0 \in$ $N(C)$. Let $\left\{y_{m}\right\}$ be a dense sequence in $S_{Y}$. Given $n \in \mathbb{N}$, define $\varphi_{m}(x)=f_{n}(x)\left(y_{m}\right)$ for every $m \in \mathbb{N}$ and $x \in C$. Then $\varphi_{m}$ are Lipschitz real-valued functions on $C$. Since $\left\{x_{n}\right\}$ is a sequence which consists of linearly independent vectors in $N(C)$ and $X_{n}=\operatorname{span}\left\{x_{k}: k \leq n\right\}$, we obtain that $\operatorname{span}\left(N(C) \cap X_{n}\right)=X_{n}$, and hence, $N(C) \cap X_{n}$ is nonempty open in $X_{n}$ (see also p.p, 9 in [11]).

Applying Lemma 7 to $X_{n}$ and $N(C) \cap X_{n}$, then $\varphi_{m}$ are Gâteaux differentiable off a null set of $N(C) \cap X_{n}$. Let $D_{\varphi_{m}}(x)$ denote the derivative of $\varphi_{m}$ at $x$.

Consider the set

$$
\begin{aligned}
W & =\left\{x \in N(C) \cap X_{n}: D_{\varphi_{m}}(x) \text { exists for every } m\right. \\
& \in \mathbb{N}\} .
\end{aligned}
$$

Then $W$ is not null set in $N(C) \cap X_{n}$. Choose $x \in W$; then the following limits exists:

$$
D_{\varphi_{m}}(x)(h)=\lim _{\lambda \longrightarrow 0} \frac{\left(f_{n}(x+\lambda h)-f_{n}(x)\right)\left(y_{m}\right)}{\lambda},
$$

for every $m \in \mathbb{N}, h \in X_{n}$. Since $f$ is Lipschitz, $\left(f_{n}(x+\lambda h)-\right.$ $\left.f_{n}(x)\right) / \lambda$ is a bounded $\omega^{*}$-Cauchy sequence when $\lambda \longrightarrow 0$. By Alaoglu theorem, $\omega^{*}-\lim _{\lambda \rightarrow 0}\left(\left(f_{n}(x+\lambda h)-f_{n}(x)\right) / \lambda\right)$ exists in $Y^{*}$. Since $D_{\varphi_{m}}(x)$ is linear for every $m \in \mathbb{N}$, the limit 
$\omega^{*}-\lim _{\lambda \longrightarrow 0}\left(\left(f_{n}(x+\lambda h)-f_{n}(x)\right) / \lambda\right)$ is linear, and hence it is a bounded linear operator from $X_{n}$ into $Y^{*}$ because $f$ is Lipschitz. Therefore, $D_{f_{n}}^{*}(x)$ exists for every $n \in \mathbb{N}$ and $x \in W$.

(ii). For every $n \in \mathbb{N}, h \in X_{n}$ and $x \in W$, we have

$$
\begin{aligned}
\left\|D_{f_{n}}^{*}(x)(h)\right\| & =\sup _{m \in N}\left|D_{f_{n}}^{*}(x)(h)\left(y_{m}\right)\right| \\
& =\sup _{m \in N}\left|\lim _{\lambda \rightarrow 0} \frac{\left(f_{n}(x+\lambda h)-f_{n}(x)\right)\left(y_{m}\right)}{\lambda}\right| \\
& \leq K\|h\| .
\end{aligned}
$$

This completes our assertion (ii).

(iii). We omit the proof because the proof of assertion (iii) is similar to the proof of assertion (c) of Theorem 14.2.18, p. 385 in [8].

Proof of Theorem 2. Without loss of generality, we assume that $0 \in N(C)$. By Theorem 2.6 in [7], $\operatorname{span}(C)=$ $\operatorname{span}(N(C))=\bigcup_{n=1}^{\infty} n N(C)$ is a dense subspace of $X$. Since $X$ is separable, there exists a sequence $\left\{x_{n}\right\}$ of linearly independent vectors in $N(C)$ such that $\operatorname{span}\left\{x_{n}\right\}$ is dense in $X$. For each $n \in N$, set $X_{n}=\operatorname{span}\left\{x_{k}: k \leq n\right\}$. Let

$$
\begin{aligned}
& D_{n}=\{x \in N(C): \\
& \quad \omega^{*}-\lim _{\lambda \rightarrow 0} \frac{f(x+\lambda h)-f(x)}{\lambda} \text { exists for every } h \\
& \left.\quad \in X_{n}\right\} .
\end{aligned}
$$

and let $D=\cap D_{n}$. Since $D_{n}$ is a Borel set, so is $D$. Therefore, for each $x \in D$,

$$
T_{x}(h)=\omega^{*}-\lim _{\lambda \rightarrow 0} \frac{f(x+\lambda h)-f(x)}{\lambda}, \quad h \in \operatorname{span}\left\{x_{n}\right\}
$$

defines a bounded linear operator from $\operatorname{span}\left\{x_{n}\right\}$ to $Y^{*}$.

Since $\operatorname{span}\left\{x_{n}\right\}$ is dense in $X$, there exists a unique bounded linear extension $T$ of $T_{x}$ from $\operatorname{span}\left\{x_{n}\right\}$ to $X$. Therefore, $T=D_{f}^{*}(x)$ and $D$ is just the set of all $\omega^{*}$-Gâteaux differentiability points of $f$ in $N(C)$. It remains to show that $\mathrm{D}$ is not null.

By Theorem 10, $\left(N(C) \backslash D_{n}\right) \cap X_{n}$ is a null set in $N(C) \cap X_{n}$. Given $z \in X$, we define $f_{z}$ by $f_{z}(x)=f(x-z), x \in(N(C)+$ $z) \cap X_{n}$. Then $f_{z}$ is a Lipschitz mapping on set $(N(C)+z) \cap X_{n}$, and by Lemma 7, $\left(N(C) \backslash D_{n}+z\right) \cap X_{n}$ is the set of all nonGâteaux differentiability points of $f_{z}$. By Lemma $8, N(C) \backslash$ $D_{n} \in \Lambda\left(\left\{x_{k}: k \leq n\right\}\right)$. Therefore, $N(C) \backslash D \in \Lambda\left(\left\{x_{n}\right\}\right)$.

Next, for any sequence $\left\{x_{n}^{\prime}\right\}$ of non-zero vectors in $X$ whose linear span is dense in $X$, we shall prove $N(C) \backslash D \in$ $\Lambda\left(\left\{x_{n}^{\prime}\right\}\right)$. If $\operatorname{span}\left\{x_{n}^{\prime}\right\} \subset \operatorname{span}(C)$, we just repeat the procedure above. Otherwise, for any $x \in\left\{x_{n}^{\prime}\right\}$ such that $x \notin \operatorname{span}(C)$, if $(N(C) \backslash D) \cap(z+R x) \neq \emptyset$ for every $z \in X$, then there exists $z^{\prime} \in N(C) \backslash D$ such that $(N(C) \backslash D) \cap(z+R x)=$ $(N(C) \backslash D) \cap\left(z^{\prime}+R x\right)=\left\{z^{\prime}\right\}$. Therefore, $N(C) \backslash D \in \Lambda(x)$. This implies that $N(C) \backslash D$ is null set.

\section{Fixed Point Property for Isometries}

In this section we apply $\omega^{*}$-Gâteaux differentiability of Lipschitz mappings to the fixed point theory. As a result, we obtain Theorem 3.

Let $C$ be a nonempty bounded closed convex subset of a Banach space $X$. Recall that a mapping $T: C \longrightarrow C$ is said to be isometry if

$$
\|T(x)-T(y)\|=\|x-y\|,
$$

whenever $x, y \in C$. We say that $C$ has the fixed point property for isometries if every isometry $T: C \longrightarrow C$ has a fixed point.

Definition 11. Suppose that $C$ is a subset of a Banach space $X$ and that $Y$ is a Banach space. We say that $C$ Lipschitz embeds into $Y^{*}$ provided that there is a mapping $f: C \longrightarrow Y^{*}$ and constants $k, K>0$ such that for all $x, y \in C$,

$$
k\|x-y\| \leq\|f(x)-f(y)\| \leq K\|x-y\| .
$$

In this case, $f$ is said to be Lipschitz embedding and the smallest possible constant $K$ is called the Lipschitz constant of the mapping, in short, $\operatorname{Lip}(f)=K$.

Proposition 12. Suppose that $C$ is a closed convex separable set in a Banach space $X$. Then $N(C) \neq \emptyset$ in $X_{C}$.

Proof. We may assume that $0 \in C$. Since $C$ is separable, $X_{C}$ is a separable space. It follows immediately from Proposition 1 in $[10]$ that $N(C) \neq \emptyset$.

Theorem 13. Suppose that $C$ is a separable closed convex set of a Banach space $X$, and that $Y$ is a separable Banach space. Then $C$ Lipschitz embeds into $Y^{*}$ if and only if $X_{C}$ is linearly isomorphic to a subspace of $Y^{*}$.

Proof.

Sufficiency. It is clearly trivial.

Necessity. From Proposition $12, N(C) \neq \emptyset$ in $X_{C}$. We may assume that $0 \in N(C)$. It follows from Theorem 2.6 in [7] that $C_{0}=\bigcup_{n=1}^{\infty} n C=\operatorname{span}(C)$ is a dense subspace of $X_{C}$. Note that $X_{C}$ is separable, thus there exists a sequence $\left\{x_{n}\right\}$ of linearly independent vectors in $N(C)$ such that $\operatorname{span}\left\{x_{n}\right\}$ is dense in $X_{C}$. Let $f: C \longrightarrow Y^{*}$ be a Lipschitz embedding. For each $n \in N$, set $X_{n}=\operatorname{span}\left\{x_{k}: k \leq n\right\}$. Consider the set of all points $x \in N(C)$ for which there is a bounded linear operator $T_{n}: X_{n} \longrightarrow Y^{*}$ such that

$$
\begin{aligned}
\lim _{\lambda \rightarrow 0} \frac{(f(x+\lambda h)-f(x))(y)}{\lambda}=T_{n}(h)(y), & \\
& h \in X_{n}, y \in Y,
\end{aligned}
$$

and

$$
k\|h\| \leq\left\|T_{n}(h)\right\|, \quad h \in X_{n}
$$

From the proof of Theorem 10, the conclusion follows using argument similar to the proof of Theorem 2 . 
Proposition 14. Suppose that $C$ is a separable closed convex set of a Banach space $X$, and that $Y$ is a dual Banach space. Then $C$ Lipschitz embeds into $Y$ if and only if $X_{C}$ is linearly isomorphic to a subspace of $Y$.

Proof. It suffices to show the necessity. Let $Y=Z^{*}$ and let $f: C \longrightarrow Z^{*}$ be a Lipschitz embedding. Since $C$ is a separable, $X_{f(C)}$ is a separable subspace of $Z^{*}$. By Corollary 14.2.22 in [8], we can find a separable Banach space $W \subset Z$ and linearly isometric embeddings $i_{1}: X_{f(C)} \longrightarrow W^{*}$ and $i_{2}: W^{*} \longrightarrow$ $Z^{*}$. Hence, $i_{1} f: C \longrightarrow W^{*}$ is a Lipschitz embedding. By Theorem $13, X_{C}$ is linearly isomorphic to a subspace of $W^{*}$. Note that $W^{*}$ is linearly isometric isomorphic to a subspace of $Z^{*}$. Therefore, $X_{C}$ is linearly isomorphic to a subspace of $Y$.

Proof of Theorem 3. Let $C$ be a nonempty bounded closed convex subset of a Banach space $X$ and let $C$ be Lipschitz embedded into a super reflexive space $Y$. We still assume $0 \epsilon$ $C$. We can also assume that $C$ is separable because the fixed point property for isometries of a bounded closed convex set in a Banach space is separably determined. Superreflexivity of $Y$ entails $Y=Y^{* *}$ and hence $Y^{* *}$ is also a super reflexive space. By Proposition 14, $X_{C}$ is linearly isomorphic to a closed subspace of $Y^{* *}$. This implies $X_{C}$ is a super reflexive space. Therefore, $C$ has the fixed point property for isometries by Maurey's fixed point theorem [12].

Remark 15. The converse Theorem 3 does not hold. In fact, for a nonempty uniformly convexifiable set $C$ of Banach space $X, C$ has the fixed point property for isometries (See [13, Theorem 4.2]). However, Beauzamy [14] proved that there exists a uniformly convexifiable set in a Banach space which can not linearly embedded into a super reflexive space.

\section{Fixed Point Property for Continuous Affine Mappings}

In this section, with the application of Baudier-LancienSchlumprecht's theorem, we obtain Theorem 4.

Let $C$ be a nonempty bounded closed convex subset of a Banach space $X$. Recall that a mapping $T: C \longrightarrow C$ is said to be affine if

$$
T(\lambda x+(1-\lambda y))=\lambda T(x)+(1-\lambda) T(y)
$$

whenever $x, y \in C$ and $\lambda \in[0,1]$. We say that $C$ has the fixed point property for continuous affine mappings if every continuous affine mapping $T: C \longrightarrow C$ has a fixed point.

Let $X$ and $Y$ be two Banach spaces and $C$ be a subset of $X$, and let $f: C \longrightarrow Y$ be a mapping. Set

$$
\rho_{f}(t)=\sup \left\{\|f(x)-f(y)\|_{Y}:\|x-y\|_{X} \geq t\right\},
$$

and

$$
\omega_{f}(t)=\sup \left\{\|f(x)-f(y)\|_{Y}:\|x-y\|_{X} \leq t\right\} .
$$

We say that $f$ is uniformly continuous if $\lim _{t \rightarrow 0} \omega_{f}(t)=0$ and $\rho_{f}(t)>0$ for all $t>0$. $C$ is said to be uniformly embedded into $Y$ provided that there is a mapping $f: C \longrightarrow Y$ such that $f$ is injective and both $f$ and $f^{-1}$ are uniformly continuous.

Proposition 16. Suppose that $C$ is a nonempty bounded closed convex subset of a Banach space $X$. If $C$ affinely uniformly embeds into a reflexive space $Y$, then $C$ has the fixed point property for continuous affine mappings.

Proof. Suppose that $C$ affinely uniformly embeds into a reflexive space $Y$. Then there exist an affinely uniformly continuous mapping $T: \mathrm{C} \longrightarrow Y$. We may assume that $0 \in C$. Defining a mapping $T_{1}(x)=T(x)-T(0)$ for all $x \in \overline{\operatorname{span}}(C)$ is a linearly uniformly continuous mapping from $\overline{\operatorname{span}}(C)$ to $Y$. Thus, $\overline{\operatorname{span}}(C)$ is a reflexive space. It follows from SchauderTychonoff theorem [15] that $C$ has the fixed point property for continuous affine mappings.

Remark 17. The affinity in Proposition 16 is necessary. Mazur [16] proved that the unit ball $B_{L_{2}}$ of $L_{2}$ is uniformly homomorphic onto the unit ball $B_{L_{1}}$ of $L_{1}$. It is easy to see that $B_{L_{2}}$ has the fixed point property for continuous affine mappings by Schauder-Tychonoff theorem [15] and $B_{L_{1}}$ does not have the fixed point property for continuous affine mappings by Theorems 3.2 in [17]. On the other hand, the Mazur theorem [16] also implies that weak compactness is usually not preserved under uniform embeddings.

Recall the construction of the Tsirelson spaces $T^{*}$ and $T$ originally designed by Tsirelson [18]. Let $E, F \subset \mathbb{N}$ and $n \in \mathbb{N}$. We denote $E<F$ if $\max (E)<\min (F)$ and $n \leq E$ if $n \leq$ $\min (E)$. Here we set $\max (\emptyset)=0$ and $\min (\emptyset)=\infty$. We say that a sequence $\left\{E_{n}\right\}_{j=1}^{n} \subset \mathbb{N}$ is admissible if $n \leq E_{1}<E_{2}<$ $\cdots<E_{n}$. Let $\left\{e_{j}\right\}$ be the canonical basis of $c_{00}$. For every $x=$ $\sum_{j=1}^{\infty} \alpha_{j} e_{j} \in c_{00}$, we put $\|x\|_{0}=\|x\|_{\mathcal{C}_{0}}=\max _{j \in N}\left|\alpha_{j}\right|$ and then define inductively for $k=1,2, \cdots$

$$
\begin{aligned}
& \|x\|_{k}=\max \left(\|x\|_{k-1}, \frac{1}{2}\right. \\
& \left.\cdot \max \left\{\sum_{j=1}^{n}\left\|E_{j}(x)\right\|_{k-1}:\left\{E_{n}\right\}_{j=1}^{n} \text { is admissible }\right\}\right) .
\end{aligned}
$$

Put

$$
\|x\|=\lim _{k \longrightarrow \infty}\|x\|_{k}=\max _{k \in \mathbb{N}}\|x\|_{k} .
$$

Then $\|\cdot\|$ a norm on $c_{00}$ and $T$ is defined to be the completion of $c_{00}$ with respect to the norm $\|\cdot\|$. We denote the dual of $T$ by $T^{*}$ which is nowadays usually referred to as $T^{*}$. For more detail, we refer the reader to [19].

For any infinite subset $M$ of $\mathbb{N}$, let $[M]^{\omega}=\{A \subset M$ : $A$ is infinite $\}$. For each $k \in \mathbb{N}$, let $[M]^{k}=\{A \subset M:|A|=k\}$, where $|A|$ denotes the cardinality of the set $A$. Elements of $[\mathbb{N}]^{k}$ will always be listed in an increasing order; i.e., for every 
$\bar{m}=\left\{m_{1}, m_{2}, \ldots, m_{k}\right\} \in[M]^{k}$, we assume that $m_{1}<m_{2}<$ $\cdots<m_{k}$. Recall that the Hamming metric is defined by

$$
\begin{aligned}
d_{H}(\bar{m}, \bar{n})= & \left|\left\{i \in\{1,2, \ldots, \min (|\bar{m}|,|\bar{n}|)\}: m_{i} \neq m_{i}\right\}\right| \\
& +\max (|\bar{m}|,|\bar{n}|)-\min (|\bar{m}|,|\bar{n}|),
\end{aligned}
$$

where $\bar{m}, \bar{n} \in[\mathbb{N}]^{k}$. Note that the metric $d_{H}$, can be seen as the graph metric on the Hamming graph over a countable alphabet, denoted $H_{k}^{\omega}(\mathbb{N})$ or simply $H_{k}^{\omega}$, where two vertices are adjacent if they differ in exactly one coordinate. Let $h$ : $\mathbb{N} \times \mathbb{N} \longrightarrow \mathbb{N}$ be a bijective. Then the map $\phi_{k}: H_{k}^{\omega} \longrightarrow T^{*}$ defined by $\phi_{k}(\bar{n})=(1 / 2)\left\|\sum_{i=1}^{k} y_{h\left(i, n_{i}\right)}\right\|$ is a Lipschitz mapping with $\operatorname{Lip}\left(\phi_{k}\right)=1$ (see, for instant, [20]).

Proof of Theorem 4. By Schauder-Tychonoff theorem [15], it suffices to show that $C$ is weakly compact. Suppose, to the contrary, that $C$ is not weakly compact. Then, by James' theorem [21], there exist $\delta>0$ and a sequences $\left\{x_{n}\right\} \subset C$ such that for all $k \in \mathbb{N}$,

$$
d\left(\operatorname{co}\left\{x_{1}, x_{2}, \ldots, x_{k}\right\}, c o\left\{x_{k+1}, x_{k+2}, \cdots\right\}\right) \geq \delta .
$$

Choose $\bar{n} \in[\mathbb{N}]^{2 k}$. Then

$$
\left\|\frac{1}{k} \sum_{i=1}^{k} x_{n_{i}}-\frac{1}{k} \sum_{i=k+1}^{2 k} x_{n_{i}}\right\| \geq \delta
$$

which implies that

$$
\left\|\sum_{i=1}^{k} x_{n_{i}}-\sum_{i=k+1}^{2 k} x_{n_{i}}\right\| \geq k \delta .
$$

For each $\bar{n} \in H_{k}^{\omega}$, define a map $\phi_{k}: H_{k}^{\omega} \longrightarrow C$ by $\phi_{k}(\bar{n})=$ $(1 / k) \sum_{i=1}^{k} x_{n_{i}}$. Then $\phi_{k}$ is a Lipschitz mapping with $\operatorname{Lip}\left(\phi_{k}\right)=$ $2 / k$.

Let $f$ be a uniform embedding from $C$ into $T^{*}$. Then $\operatorname{Lip}\left(f \circ \phi_{k}\right) \leq \omega_{f}(2 / k)$. By Theorem 4.4 in [20], for all $k \geq 1$, there exists $M^{\prime} \in[\mathbb{N}]^{\omega}$ such that

$$
\left\|f \circ \phi_{k}(\bar{m})-f \circ \phi_{k}(\bar{n})\right\| \leq 5 \operatorname{Lip}\left(f \circ \phi_{k}\right),
$$

for all $\bar{m}, \bar{n} \in\left[M^{\prime}\right]^{k}$. This implies

$$
\left\|f \circ \phi_{k}(\bar{m})-f \circ \phi_{k}(\bar{n})\right\| \leq 5 \omega_{f}\left(\frac{2}{k}\right) .
$$

In particular, for all $k \geq 1$, choose $\bar{m}, \bar{n} \in\left[M^{\prime}\right]^{k}$ such that $m_{1}<m_{1}<\cdots<m_{k}<n_{1}<n_{2} \cdots<n_{k}$. We obtain

$$
0<\rho_{f}(\delta) \leq 5 \omega_{f}\left(\frac{2}{k}\right) .
$$

This is a contradiction when $k$ is sufficiently large and which completes our proof.

\section{Data Availability}

No data were used to support this study.

\section{Conflicts of Interest}

The authors declare that they have no conflicts of interest.

\section{Acknowledgments}

The first author was supported by Educational Commission of Hubei Province of China, Grant no. B2018046. The corresponding author was supported by NSFC, Grant no. 11501108, and by NSFF, Grant no. 2015J01579, 1991 Mathematics Subject Classification, 47H10, 46B03, and 46G05.

\section{References}

[1] N. Aronszajn, "Differentiability of Lipschitzian mappings between Banach spaces," Studia Mathematica, vol. 57, no. 2, pp. 147-190, 1976.

[2] J. P. Christensen, "Measure theoretic zero sets in infinite dimensional spaces and applications to differentiability of Lipschitz mappings," in Proceedings of the Second Colloquium of Functional Analysis of Bordeaux, vol. 10, pp. 29-39, Publications du Département de Mathématiques de Lyon, Université de Lyon, Lyon, France, 1973.

[3] Y. Benyamini and J. Lindenstrauss, "Geometric nonlinear functional analysis, Colloquium publications," American Mathematical Society, vol. 48, 2000.

[4] M. Csörnyei, "Aronszajn null and Gaussian null sets coincide," Israel Journal of Mathematics, vol. 111, pp. 191-202, 1999.

[5] R. R. Phelps, "Gaussian null sets and differentiability of Lipschitz map on Banach spaces," Pacific Journal of Mathematics, vol. 77, no. 2, pp. 523-531, 1978.

[6] P. Mankiewicz, "On the differentiability of Lipschitz mappings in Fr chet spaces," Studia Mathematica, vol. 45, pp. 15-29, 1973.

[7] L. Cheng and W. Zhang, "A note on nonsupport points, negligible sets, Gateaux differentiability and Lipschitz embeddings," Journal of Mathematical Analysis and Applications, vol. 350, no. 2, pp. 531-536, 2009.

[8] F. Albiac and N. J. Kalton, Topics in Banach Space Theory, vol. 233 of Graduate Texts in Mathematics, Springer, New York, NY, USA, 2nd edition, 2016.

[9] S. Heinrich and P. Mankiewicz, "Applications of ultrapowers to the uniform and Lipschitz classification of banach spaces," Studia Mathematica, vol. 73, no. 3, pp. 225-251, 1982.

[10] J. Rainwater, "Yet more on the differentiability of convex functions," Proceedings of the American Mathematical Society, vol. 103, no. 3, pp. 773-778, 1988.

[11] R. B. Holmes, Geometric Functional Analysis and Its Applications, Graduate Texts in Mathematics, Springer, New York, NY, USA, 1975.

[12] B. Maurey, "Points fixe des contractions sur un convexe forme de L1, in Seminaire d'Analyse Fonctionnelle," Vol. 80-81, Exposé No. VIII, Ecole Normale Polytechnique, Palaiseau, 1981.

[13] L. Cheng, Q. Cheng, and J. Zhang, "On super fixed point property and super weak compactness of convex subsets in Banach spaces," Journal of Mathematical Analysis and Applications, vol. 428, no. 2, pp. 1209-1224, 2015.

[14] B. Beauzamy, "Opérateurs uniformément convexifiants," Studia Mathematica, vol. 57, no. 2, pp. 103-139, 1976.

[15] J. Dugundji and A. Granas, Fixed Point Theory, P.W.N., Warszawa, 1982. 
[16] S. Mazur, "Une remarque sur l'homéomorphie des champs fonctionnels," Studia Mathematica, vol. 1, no. 1, pp. 83-85, 1930.

[17] T. D. Benavides, M. A. Japon Pineda, and S. Prus, "Weak compactness and fixed point property for affine mappings," Journal of Functional Analysis, vol. 209, no. 1, pp. 1-15, 2004.

[18] B. S. Tsirel'son, "It is impossible to imbed $l_{p}$ of $c_{0}$ into an arbitrary Banach space," Funkcional. Anal. i Prilozen, vol. 8, no. 2, pp. 57-60, 1974.

[19] T. Figiel and W. B. Johnson, "A uniformly convex Banach space which contains no l, " Compositio Mathematica, vol. 29, pp.179190, 1974.

[20] F. Baudier, G. Lancien, and T. Schlumprecht, "The coarse geometry of Tsirelson's space and applications," Journal of the American Mathematical Society, vol. 31, no. 3, pp. 699-717, 2018.

[21] R. C. James, "Weakly compact sets," Transactions of the American Mathematical Society, vol. 113, pp. 129-140, 1964. 


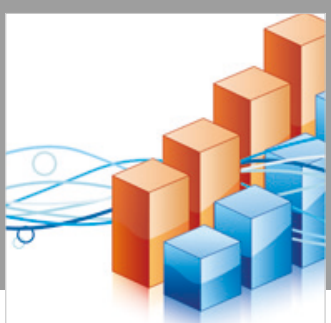

Advances in

Operations Research

\section{-n-m}
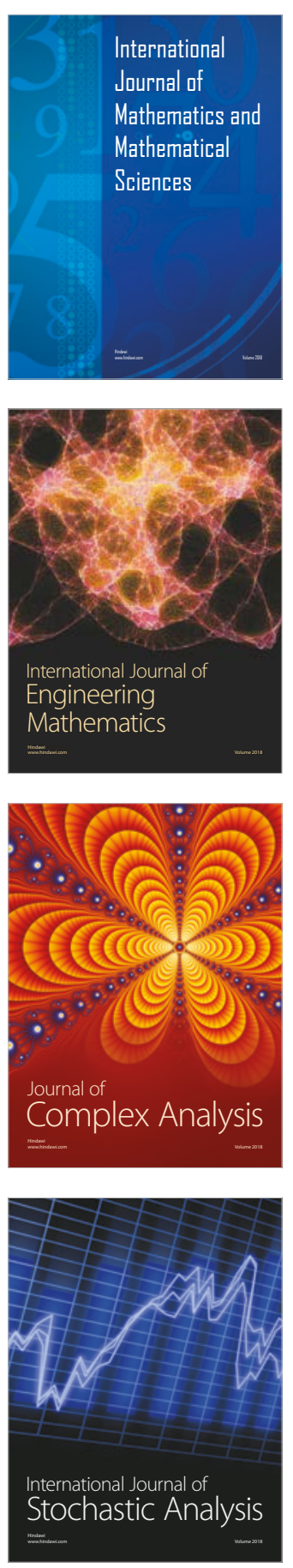
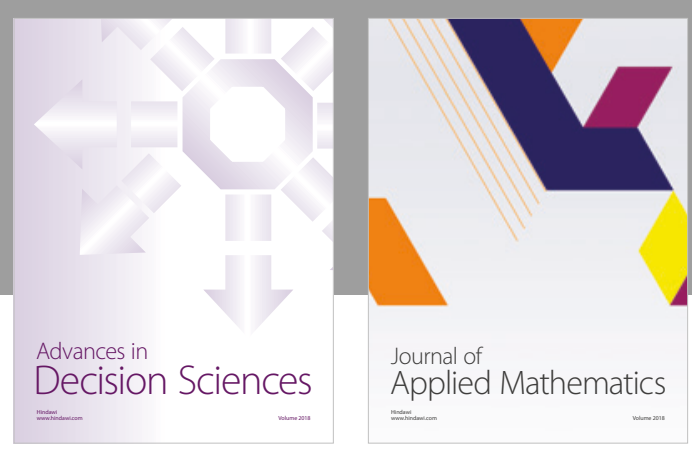

Journal of

Applied Mathematics
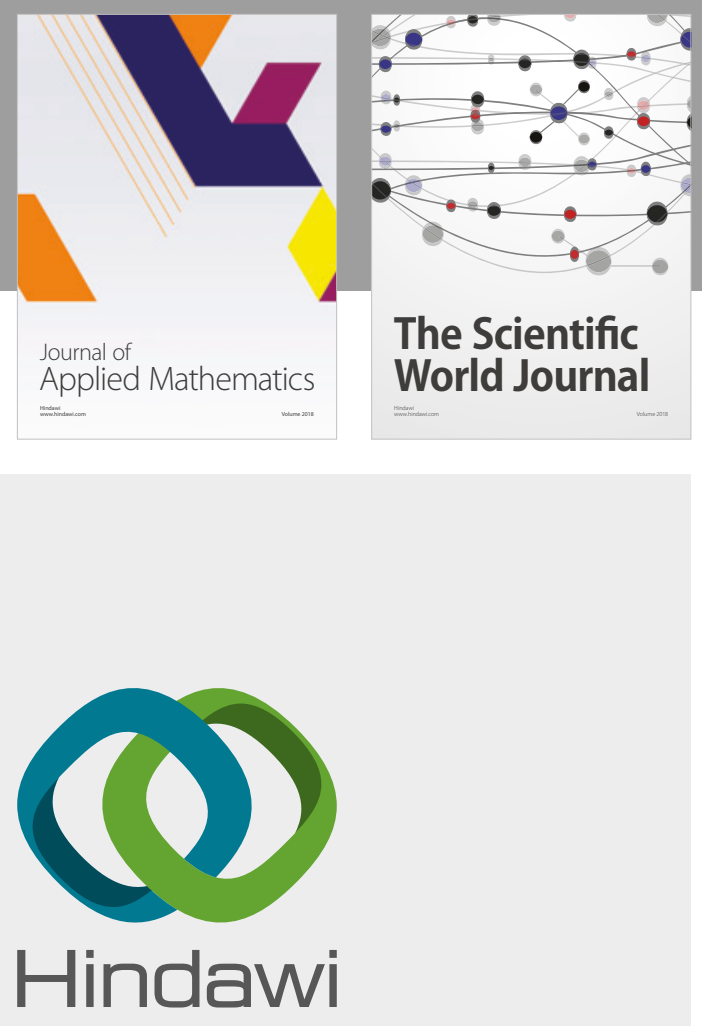

Submit your manuscripts at

www.hindawi.com

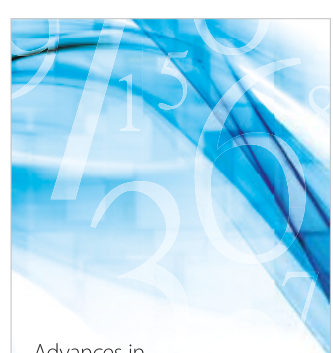

Advances in
Numerical Analysis
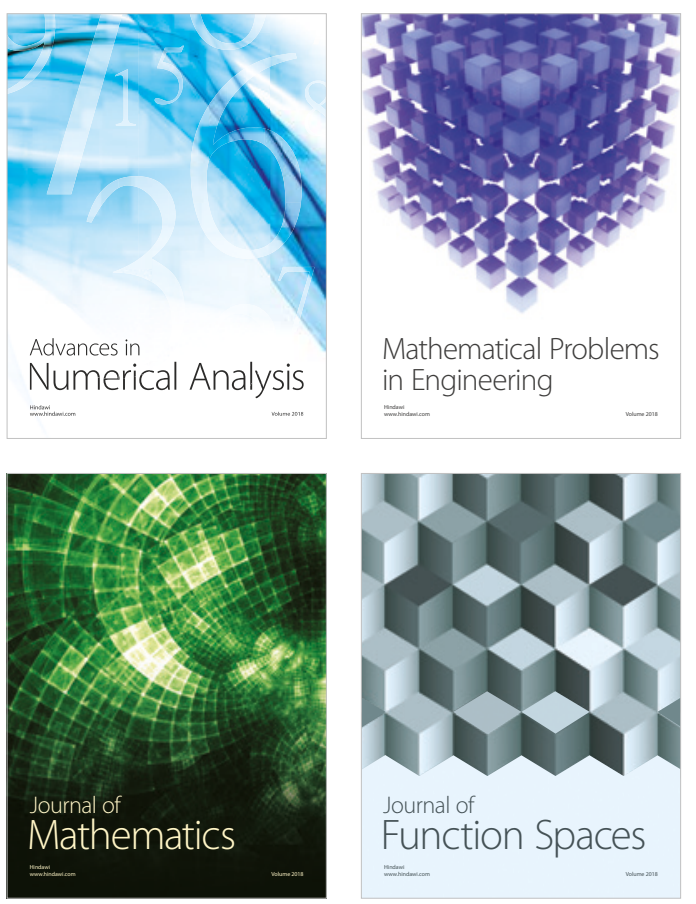

Mathematical Problems in Engineering

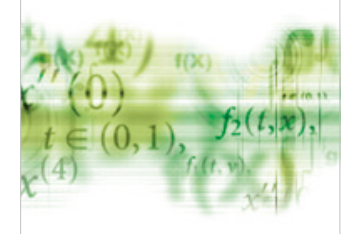

International Journal of

Differential Equations

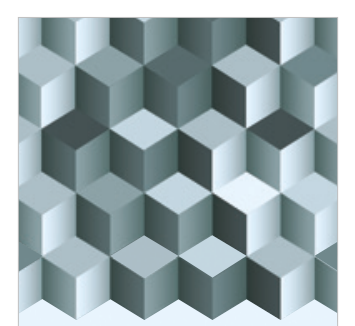

Journal of

Function Spaces
The Scientific

World Journal

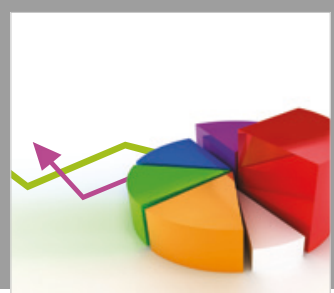

Journal of

Probability and Statistics
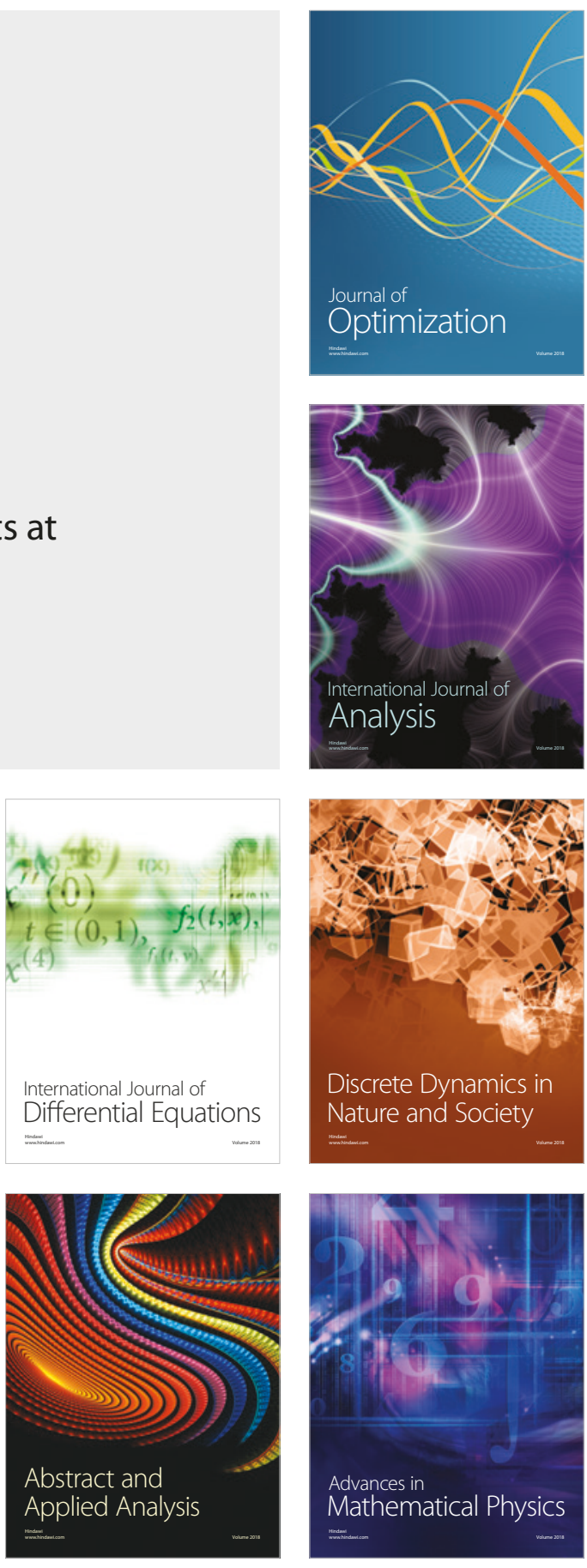